\title{
Robust PID Controller Design for a Real Electromechanical Actuator
}

\author{
Rafik Salloum \\ Control Department, Electrical Engineering Faculty \\ School of Railway Engineering, Iran University of Science and Technology \\ Malek-Ashtar University of Technology \\ Lavizan, Shahid Babaee, 15875-1774 Tehran, Iran \\ E-mail: rafsal@mut.ac.ir; rafsal@mail.iust.ac.ir
}

\section{Bijan Moaveni}

School of Railway Engineering, Iran University of Science and Technology

Narmak, 16846-13114 Tehran, Iran; E-mail: b_moaveni@iust.ac.ir

\section{Mohammad Reza Arvan}

Control Department, Electrical Engineering Faculty

Malek-Ashtar University of Technology

Lavizan, Shahid Babaee, 15875-1774 Tehran, Iran; E-mail: arvan@mut.ac.ir

\begin{abstract}
Electromechanical actuators (EMA's) are of interest for applications that require easy control and high dynamics. In this paper, we design a robust PID controller for position control of a real electromechanical actuator. An EMA is modeled as a linear system with parametric uncertainty by using its experimental input-output data. PID controllers are designed by graphical findings of the regions of stability with pre-specified margins and bandwidth requirements and by applying the complex Kharitonov's theorem. This novel method enables designers to make the convenient trade-off between stability and performance by choosing the proper margins and bandwidth specifications. The EMA control system is passed to the Bialas' test, and validated on the basis of meeting a desired set of specifications. The effects of parameter variations on the system's stability and performance are analyzed and the simulation and test results show that the EMA with the new controller, in addition to robustness to parametric uncertainties, has better performance compared to the original EMA control system. The simulation and test results prove the superiority of the performance of the new EMA over the original EMA control system pertaining to its robustness to parametric uncertainties.
\end{abstract}

Keywords: electromechanical actuator (EMA); robust PID controller; Kharitonov theorem 


\section{Introduction}

In recent years, electromechanical actuators (EMA's) are in high demand in robotics and aerospace science industries. An EMA has attractive characteristics such as simplicity, reliability, low cost, high dynamic characteristics, and easy control [1-3]. However, EMA modeling is subject to uncertainty due to several reasons, including operating point changes, parametric variations due to temperature changes, non-modeled dynamics, and asymmetric behavior. Consequently, the desired EMA's performance will be unachievable and, in some cases, its stability may be lost. Usually, based on experience, this problem will not be solved by using the conventional controllers; instead robust controllers are needed to obtain the desired performance and stabilization demands in dealing with dynamic uncertainties [4-6].

The primary motivation for designing the EMA was to access the ameliorated and favorable robustness to meet the application requirements. Robust controllers were designed to achieve robust stability, good tracking, and disturbance attenuation using the Lyapunov-based synthesis concept in [7, 8]. A genetic optimized PID controller was designed in [9], in order to improve the EMA system transient state behavior. A robust $\mathrm{H}_{\infty}$ controller for an EMA, was designed and tested to achieve a faster and more accurate system in [10].

There is a wide range of applications for PID controllers in industry due to their simplicity and effectiveness. However, the tuning process, whereby, the proper values for the controller parameters are obtained is a critical challenge. Also, the traditional PID controller lacks robustness against large system parameter uncertainties, the reason lies in the insufficient number of parameters to deal with the independent specifications of time-domain response, such as, settling time and overshooting [11]. Much effort is involved in designing robust PI, PD, or PID controllers for uncertain systems, based on different robust design methods, known in literature as Kharitonov's Theorem, Small Gain Theorem, $\mathrm{H}_{\infty}$ and Edge Theorem [12-14]. A graphical design method of tuning the PI and PD controllers achieving gain and phase margins is developed in [15]. An approach to design PID controllers for systems without time delay was presented in [16].

In this paper, a novel approach to the design of a robust PID controller for an EMA system with time delay, is proposed and applied to a "motor with harmonic drive" subsystem of the EMA system. This approach is presented, based on the complex Kharitonov theorem for interval model with time delay. The applied method enables the designer to make the convenient trade-off between stability and performance by choosing the appropriate margins and bandwidth specifications. The closed loop EMA system and "motor with harmonic drive" subsystem is modeled as linear systems with parametric uncertainty. The modeling is accomplished, by identifying the EMA system and "motor with harmonic drive" subsystem using experimental input-output data in different 
operating points. Bialas' test based on edge theorem is also applied, to emphasize the robust controller validity.

The robust controller design procedure is then studied under simulation conditions, and its effectiveness is proven by comparing the closed loop performance identified through test data with that achieved by using the robust PID controller.

After presenting this introduction, we discuss the following: The EMA uncertain model and experimental set-up as described in Section 2. In Section 3, the robust PID computations by Kharitonov's theorem with bandwidth, phase and gain margins constraints are explained. In Section 4, the design validation is carried out and finally, conclusion are drawn.

\section{Experimental Set-up and Uncertainty Modeling}

EMA mainly consists of a DC motor driven by PWM driver, a harmonic drive reducer, a potentiometer position sensor, and a controller as shown in Figure 1. In this figure, $r$ is the setpoint voltage, $v$ is the PWM output, and $\delta$ is the output angle.

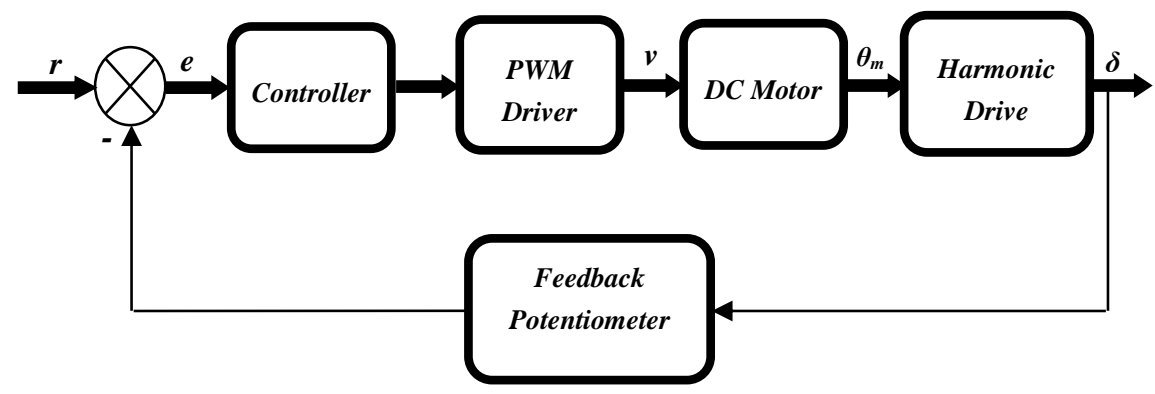

Figure 1

EMA block diagram

The experimental set-up consists of a permanent magnet DC motor with integrated harmonic drive gearing with 300:1 reduction ratio. The motor is driven by a PWM driver. The actual shaft position is recorded by a potentiometer fixed on the output shaft. A data acquisition card, which is connected to a PC, records the readings from the potentiometer.

The model of the DC motor and position control is well known in the literature $[17,18]$. The model of the subsystem under consideration (motor with harmonic drive reducer) is:

$\frac{\delta(s)}{v(s)}=\frac{K_{m} / N}{s\left(L J_{m} s^{2}+\left(L b+R J_{m}\right) s+R b+K_{m}^{2}\right)}$ 
where $K_{m}(\mathrm{~N} . \mathrm{m} / \mathrm{A})$ is the motor's torque constant, $L(\mathrm{H})$ and $R(\mathrm{Ohm})$ are the inductance and resistance of motor control coil respectively, $b(\mathrm{~N} . \mathrm{m} / \mathrm{rad} / \mathrm{sec})$ is the viscous damping coefficient, $N$ is the harmonic drive gear ratio, and $J_{m}\left(\mathrm{Kg} . \mathrm{m}^{2}\right)$ is the rotor's moment of inertia.

Hence, considering that the change of armature current in time is negligible, we can use engineering judgment to neglect $L$. In addition, the viscous damping can be neglected. So, the EMA model can be represented as follows:

$\frac{\delta(s)}{r(s)}=\frac{K}{s^{3}+\frac{s^{2}}{\tau_{m}}+K \cdot K_{f}}$

Where $K$ is a constant corresponds to the open loop gain, controller, and motor constants. $K_{f}$ is the potentiometer coefficient in $v / d e g$, and

$$
\tau_{m}=\frac{R J_{m}}{K_{m}^{2}}
$$

In this phase, we planned to verify best fitting. To achieve this goal, a method of classic identification was used to establish the EMA's model from the test data, by minimizing the mean square error (MSE). While applying this method, five working points for the EMA system were used for model identification. The final output was the extraction of the uncertain model. For example, the test results of the studied EMA system in one working point are shown in Figure 2, whose model is:

$$
G_{1}(s)=\frac{\delta(s)}{r(s)}=\frac{1}{\left(\frac{s}{148}+1\right)\left(\frac{s^{2}}{105^{2}}+2 * 0.89 * \frac{s}{105}+1\right)}
$$

Similarly, the models corresponding to the other working points were identified, and the nominal model of the EMA system is:

$$
G_{\text {EMA.nom }}(s)=\frac{\delta(s)}{r(s)}=\frac{1}{\left(\frac{s}{140}+1\right)\left(\frac{s^{2}}{119^{2}}+2 * 0.831 * \frac{s}{119}+1\right)}
$$

Figure 3 shows the actual response curve and the identified model response.

It can be seen from Figure 4 that the frequency response of the model and the actual response of the EMA, are basically, identical. Therefore, the transfer function model described in (3), is recognized to approximately describe the original EMA system.

Next, the model of the "motor with harmonic drive" subsystem was identified for five working points. For instance, the test results in one working point of the studied EMA system are shown in Figure 4 and its model has the following form:

$$
G_{\text {HDMotor } 1}(s)=\frac{\delta(s)}{v(s)}=\frac{787.3 e^{-0.0027 s}}{\left(s^{2}+140.4 s+75.7\right)}
$$



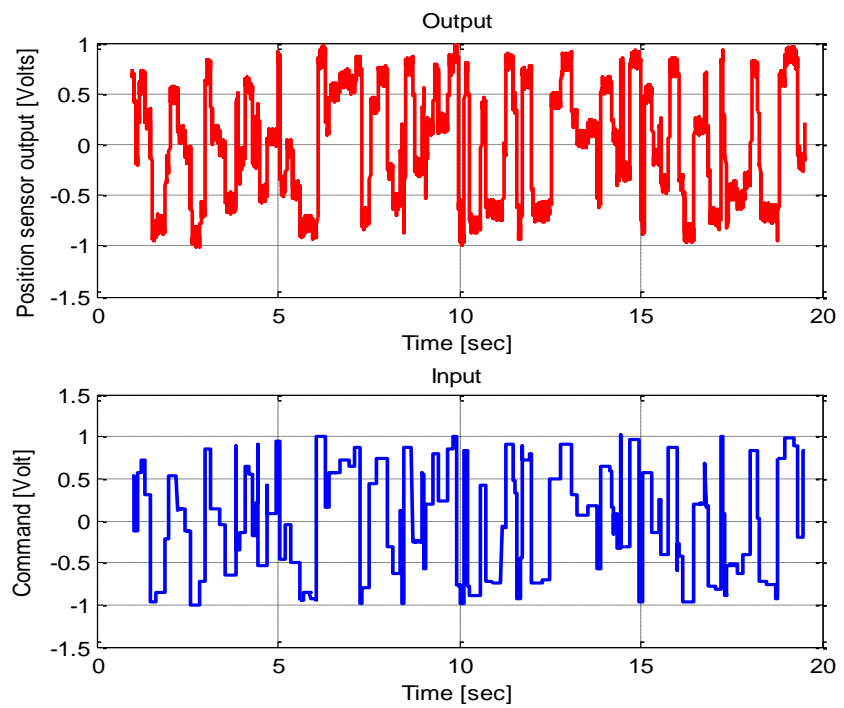

Figure 2

Input and output data captured from EMA

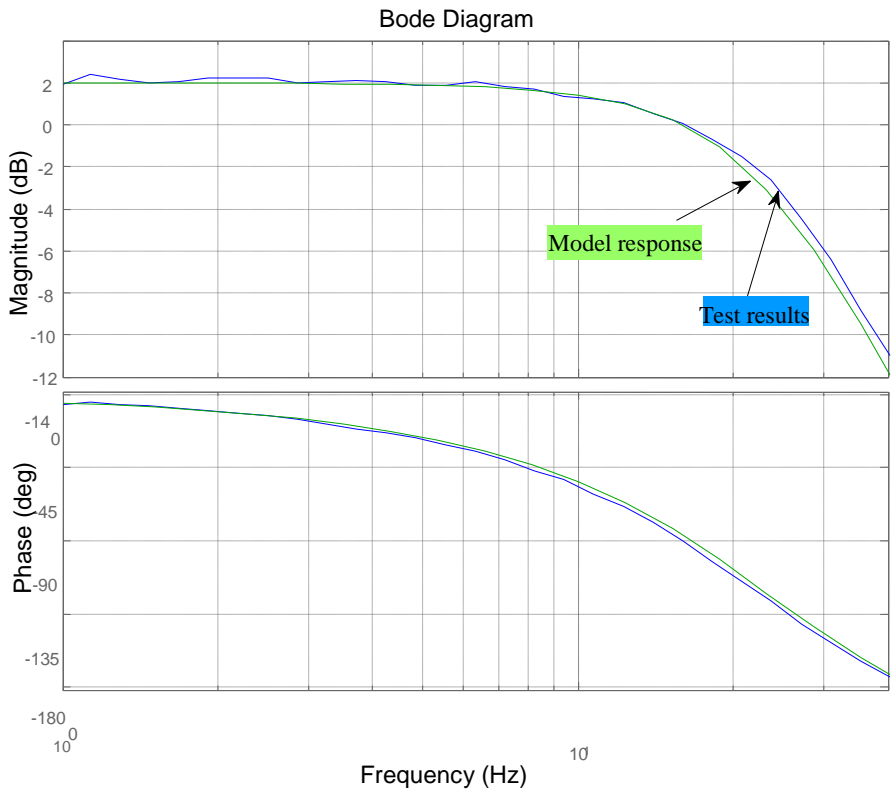

Figure 3

Frequency responses of the EMA's model and its test results 


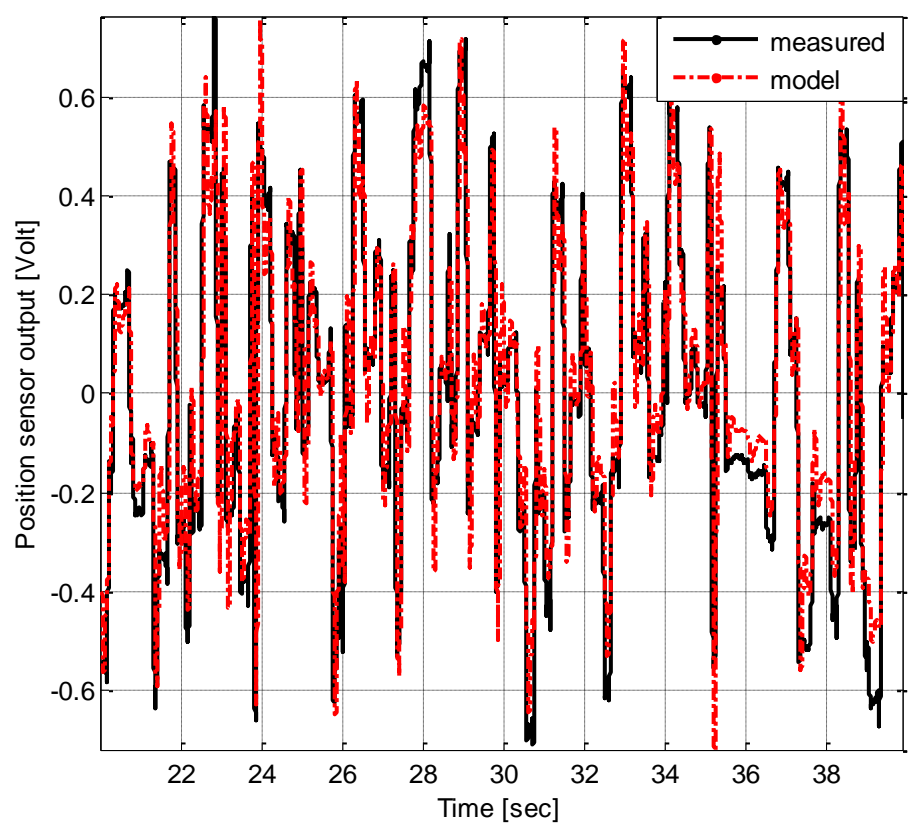

Figure 4

Outputs of "Motor and harmonic drive" subsystem and its model

The parametric uncertain model, with time delay, was built to be used in the robust controller design.

$G_{H D M o t o r}(s)=\frac{K}{\left(s^{2}+q_{1} s+q_{2}\right)} e^{-T_{d} s}$

The uncertain parameters of the model and their intervals are:

- $\quad K \in[512.3,949.7] ; K_{\text {nominal }}=750.4$

- $q_{1} \in[90.4,197.6] ; q_{1 . n o m i n a l}=144.2$

- $\quad q_{2} \in[35,90.5] ; q_{2 . n o m i n a l}=70.5$

- $T_{d} \in[1.7,3.5] ; T_{\text {d.nominal }}=2.55 \mathrm{msec}$

\section{Robust Controller Design by Kharitonov Theorem}

In this section, the Kharitonov's theorem will be briefly presented, and it will be applied to design a robust controller for the EMA system under consideration. The studied system with gain-phase margin tester is illustrated in Figure 5. 


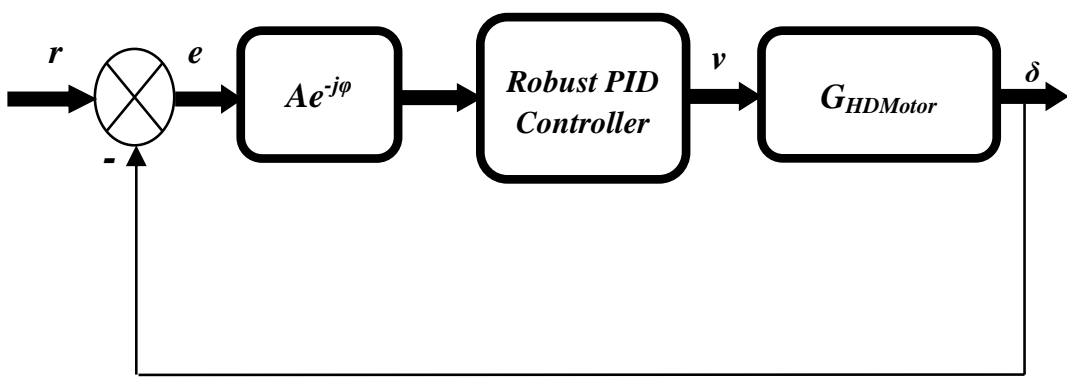

Figure 5

The studied control system with gain-phase margin tester

\subsection{Kharitonov's Theorem and Kharitonov's Rectangles}

Kharitonov's theorem deals with the stability of a system with closed-loop characteristic polynomial. Consider $I^{*}(s)$ to be the set of closed-loop characteristic polynomials of degree $n$ for the interval systems of the form:

$P(s)=p_{0}+p_{1} s+p_{2} s^{2}+\cdots+p_{n} s^{n}$

Where $p_{i}$ is a complex presented as: $p_{i}=a_{i}+j b_{i} ; a_{i} \in\left[\underline{a_{i}}, \overline{a_{i}}\right]$ and $b_{i} \in\left[\underline{b_{i}}, \overline{b_{i}}\right]$ for $i=0,1, \ldots, n$. The degree is assumed to be invariant over the polynomials family. The necessary and sufficient condition for the stability of the entire family is formulated, in the following theorems. In such a case, the set of Kharitonov systems is as follows:

$G_{K}(s)=\left\{\frac{K_{B}^{i}(s)}{K_{A}^{j}(s)}: i, j=1,2,3,4\right\}$

Where $K_{B}^{i}(s), i=1,2,3,4$ and $K_{A}^{j}(s), j=1,2,3,4 \quad$ denote the Kharitonov's polynomials associated with numerators and denumerators of the interval system respectively.

\section{Theorem 1: Kharitonov's Theorem}

Every polynomial in the family $I^{*}(s)$ is Hurwitz if and only if the following eight extreme polynomials are Hurwitz: [19]

$$
\begin{aligned}
& K^{1}(s)=\left(\underline{a_{0}}+j \underline{b_{0}}\right)+\left(\underline{a_{0}}+j \overline{b_{0}}\right) s+\left(\overline{a_{0}}+j \overline{b_{0}}\right) s^{2}+\left(\overline{a_{0}}+j \underline{b_{0}}\right) s^{3}+\cdots \\
& K^{2}(s)=\left(\overline{a_{0}}+j \overline{b_{0}}\right)+\left(\overline{a_{0}}+j \underline{b_{0}}\right) s+\left(\underline{a_{0}}+j \underline{b_{0}}\right) s^{2}+\left(\underline{a_{0}}+j \overline{b_{0}}\right) s^{3}+\cdots \\
& K^{3}(s)=\left(\overline{a_{0}}+j \underline{b_{0}}\right)+\left(\underline{a_{0}}+j \underline{b_{0}}\right) s+\left(\underline{a_{0}}+j \overline{b_{0}}\right) s^{2}+\left(\overline{a_{0}}+j \overline{b_{0}}\right) s^{3}+\cdots \\
& K^{4}(s)=\left(\underline{a_{0}}+j \overline{b_{0}}\right)+\left(\overline{a_{0}}+j \overline{b_{0}}\right) s+\left(\overline{a_{0}}+j \underline{b_{0}}\right) s^{2}+\left(\underline{a_{0}}+j \underline{b_{0}}\right) s^{3}+\cdots
\end{aligned}
$$




$$
\begin{aligned}
& K^{5}(s)=\left(\underline{a_{0}}+j \underline{b_{0}}\right)+\left(\overline{a_{0}}+j \underline{b_{0}}\right) s+\left(\overline{a_{0}}+j \overline{b_{0}}\right) s^{2}+\left(\underline{a_{0}}+j \overline{b_{0}}\right) s^{3}+\cdots \\
& K^{6}(s)=\left(\overline{a_{0}}+j \overline{b_{0}}\right)+\left(\underline{a_{0}}+j \overline{b_{0}}\right) s+\left(\underline{a_{0}}+j \underline{b_{0}}\right) s^{2}+\left(\overline{a_{0}}+j \underline{b_{0}}\right) s^{3}+\cdots \\
& K^{7}(s)=\left(\overline{a_{0}}+j \underline{b_{0}}\right)+\left(\overline{a_{0}}+j \overline{b_{0}}\right) s+\left(\underline{a_{0}}+j \overline{b_{0}}\right) s^{2}+\left(\underline{a_{0}}+j \underline{b_{0}}\right) s^{3}+\cdots \\
& K^{8}(s)=\left(\underline{a_{0}}+j \overline{b_{0}}\right)+\left(\underline{a_{0}}+j \underline{b_{0}}\right) s+\left(\overline{a_{0}}+j \underline{b_{0}}\right) s^{2}+\left(\overline{a_{0}}+j \overline{b_{0}}\right) s^{3}+\cdots
\end{aligned}
$$

\section{Theorem 2}

The closed loop system containing the interval plant $\mathbf{G}(\mathrm{s})$ is robustly stable if and only if each of the Kharitonov systems in $G_{K}(s)$ is stable. [19]

\section{Definition: Kharitonov Rectangle}

Evaluating the four Kharitonov polynomials $K^{l}(s), K^{2}(s), K^{3}(s)$, and $K^{4}(s)$ at $s=$ $j \omega_{0}$, the four vertices of Kharitonov's rectangle will be obtained. Therefore, given an interval polynomial family $P(s, \boldsymbol{q})$ and a fixed frequency $\omega=\omega_{0}$, the value $P$ $\left(j \omega_{0}, \boldsymbol{q}\right)$ is a rectangle whose vertices are given by $K_{i}\left(j \omega_{0}\right)$ for $i=1,2,3,4$. [19]

\section{Theorem 3: Origin Exclusion for Interval Families}

An interval polynomial family $P(s, \boldsymbol{q})$ has invariant degree and at least one stable member $P\left(s, q^{*}\right)$ is robustly stable, if and only if, the origin of the complex plan is excluded from the Kharitonov's rectangle at all nonnegative frequencies, i.e. $0 \notin P\left(j \omega_{0}, \boldsymbol{q}\right)$ for all frequencies. Practically, it is enough to check the zero exclusion for all $\omega \leq \omega_{c}$, i.e. for frequencies that are less than the crossover frequency. [19]

\subsection{Controller Design}

In this subsection, we design a robust PID controller, which robustly stabilizes the uncertain system, and guarantees the desired performance of closed loop system.

The required response of the EMA system to be designed, is a deadbeat response as shown in Table 1.

Table 1

System performance requirements

\begin{tabular}{|c|c|}
\hline Parameter & Value \\
\hline Rise time & $t_{r}<40 \mathrm{msec}$ \\
\hline Settling time & $t_{\mathrm{s}}<60 \mathrm{msec}$ \\
\hline Steady-state position error & 0 \\
\hline Overshoot & $<1 \%$ \\
\hline
\end{tabular}




\begin{tabular}{|c|c|}
\hline Bandwidth & $10 \mathrm{~Hz} \leq \mathrm{BW} \leq 40 \mathrm{~Hz}$ \\
\hline Gain margin & $G_{m} \geq 7 \mathrm{~dB}$ \\
\hline Phase margin & $P_{m} \geq 40^{\circ}$ \\
\hline
\end{tabular}

Since the studied system has a time delay and gain-phase margin tester, the complex Kharitonov system will be used. The closed-loop characteristic polynomial of EMA system in Figure 5 is:

$$
\begin{gathered}
P(s)=\left[s^{3}+q_{1} s^{2}+q_{2} s\right]+A \cdot K \cdot\left(K_{d} s^{2}+K_{p} s+K_{i}\right)\left(\cos \omega T_{d}-j \sin \omega T_{d}\right)(\cos \varphi \\
-j \sin \varphi)
\end{gathered}
$$

where $K_{p}, K_{i}$, and $K_{d}$ are the coefficients of the PID controller. It is clear from (8) that the polynomial has invariant degree. The coefficients of PID controller $K_{p}$, and $K_{i}$ can be expressed as functions of uncertainties, frequency, and $K_{d}$ as follows:

$K_{p}=f\left(\omega, K_{d}, a_{i}, b_{i}\right) ; K_{i}=g\left(\omega, K_{d}, a_{i}, b_{i}\right)$ for $\omega \epsilon[0, \infty]$

By varying the frequency, it is possible to draw the stability boundary in $K_{p}-K_{i}$ plan for certain $K_{d}$.

The effect of the time delay $T_{d}$ on the stabilizing region will be verified. The margins and $K_{d}$ are assigned to be as follows: $A=1, \varphi=0^{\circ}$ and $K_{d}=0.08$. The edge polynomial $K^{6}(s)$, which has the smallest stabilizing area, is illustrated in Figure 6.

The effect of the coefficient $K_{d}$ is also considered. The larger $K_{d}$ results in larger stability region as it is shown in Figure 7 for the first Kharitonov polynomial. 


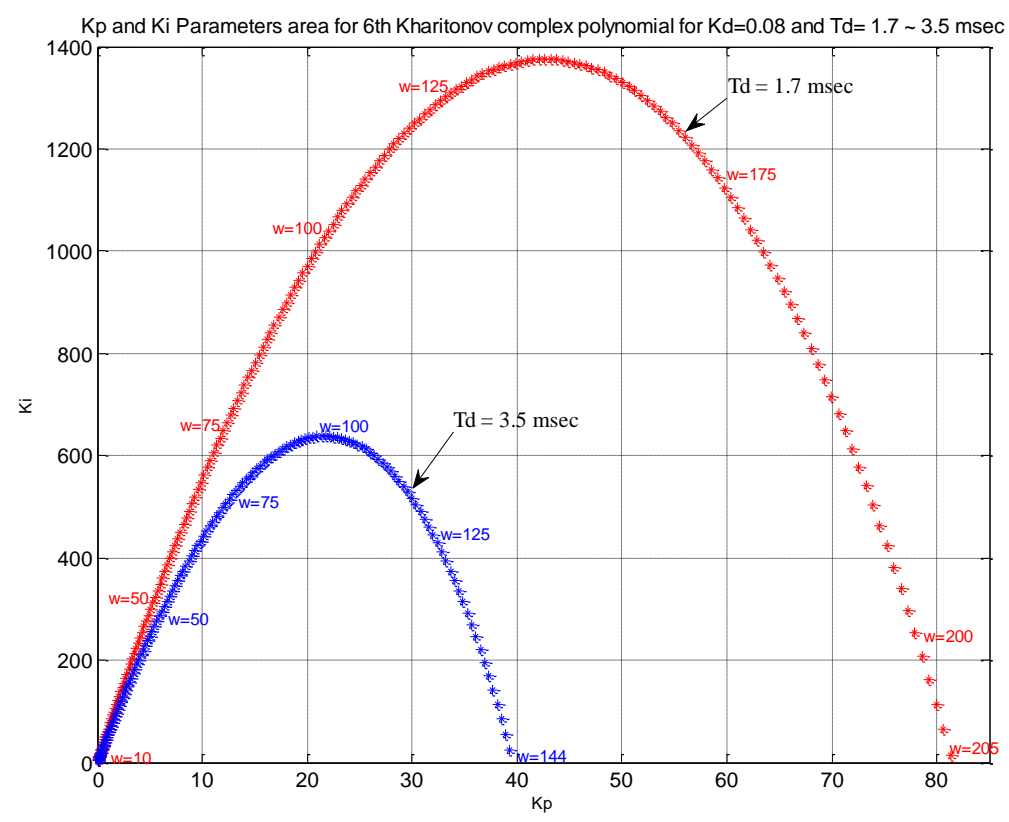

Figure 6

The effect of time delay on the stabilizing area

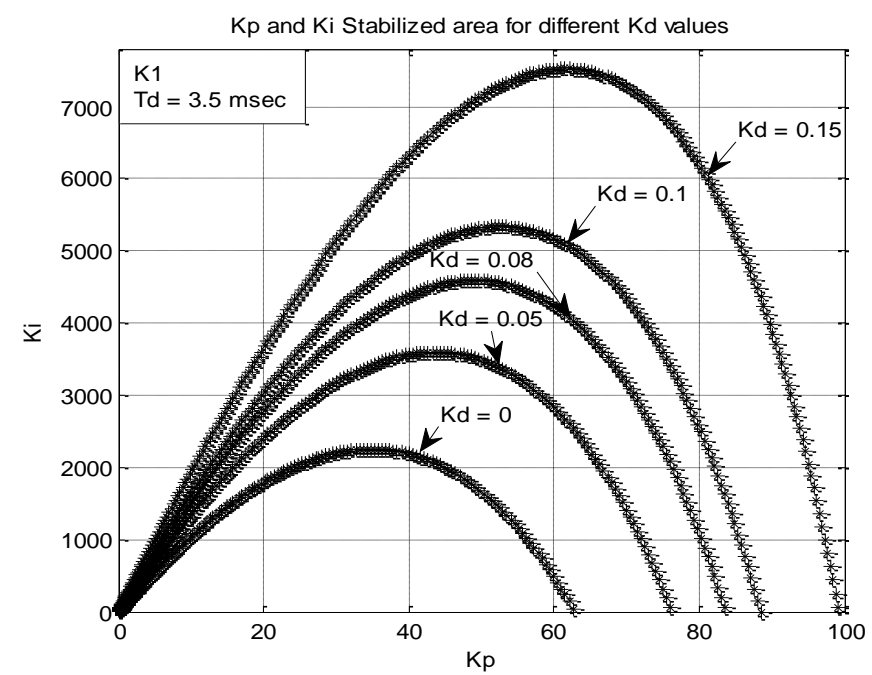

Figure 7

$K^{l}$ for different values of $K_{d}$ 
Subsequently, the phase margin constraint is the issue of consideration, $P_{m} \geq 40^{\circ}$. In this case, all eight Kharitonov's polynomials are plotted in Figure 8, and the stabilized area is that restricted under $K^{6}$ and $K^{8}$ polynomials.

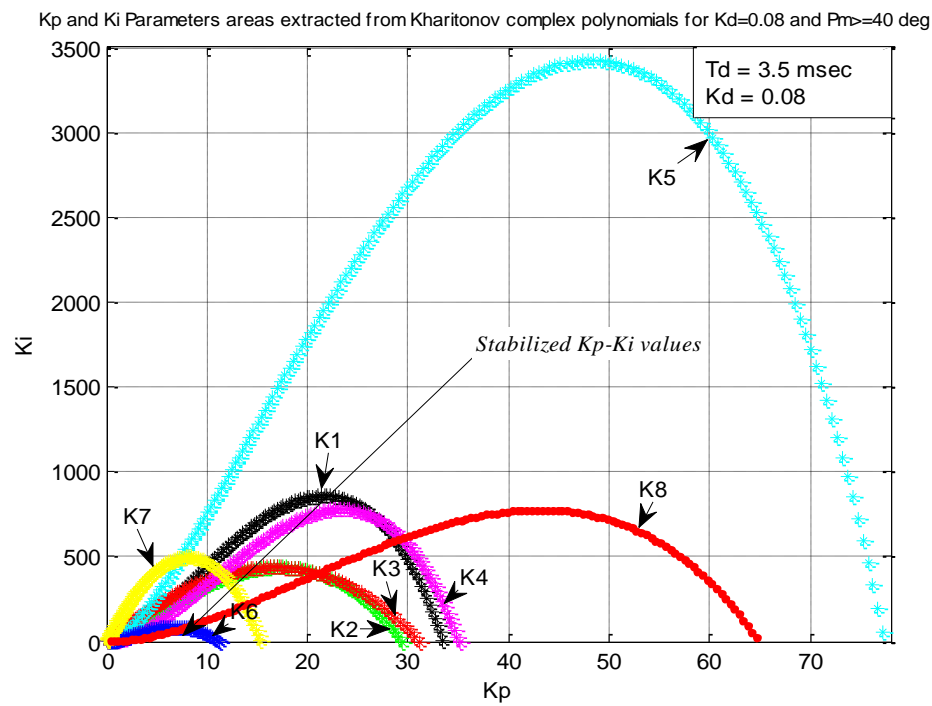

Figure 8

The stabilized lapped area for $P_{m} \geq 40^{\circ}$

The next step, in addition to the phase margin constraint, the gain margin constraint will be added $G_{m} \geq 7 d B$. The first four Kharitonov's polynomials are separately plotted in Figure 9. The lapped region under the two margins curves is the stabilizing region where the margins constraints are achieved. 

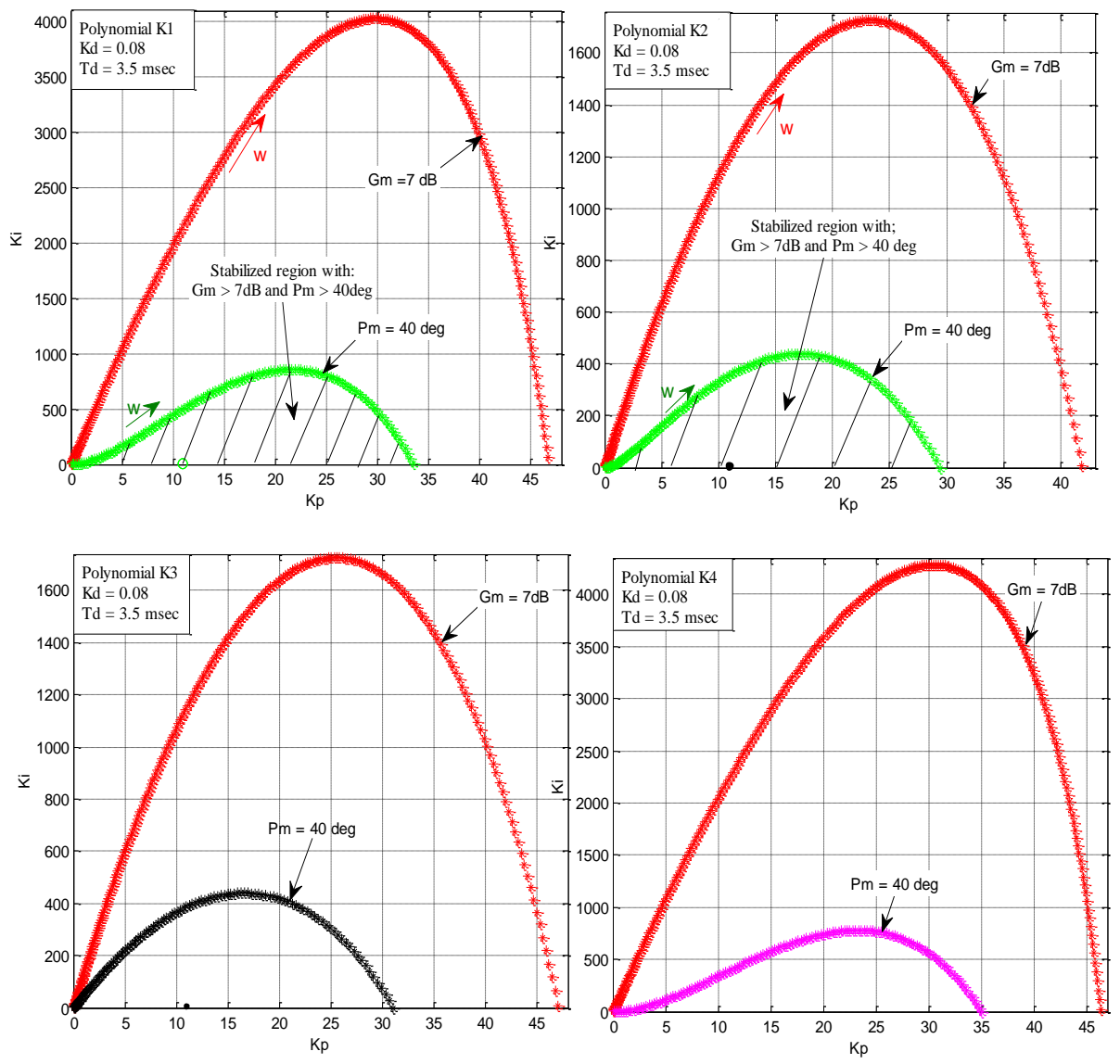

Figure 9

The stabilized lapped areas for the 4 first polynomials with $P_{m} \geq 40^{\circ}$ and $G_{m} \geq 7 \mathrm{~dB}$

Following, the bandwidth condition with upper and lower limits will lead to the restricted area shown in Figure 10 for bandwidth between 10 and $40 \mathrm{~Hz}$.

Thus, all controller's coefficients situated in the common lapped region, shown in Figure 11, robustly stabilize the closed loop EMA system over all the uncertainties under consideration, and ensure the performance margins and bandwidth conditions. 


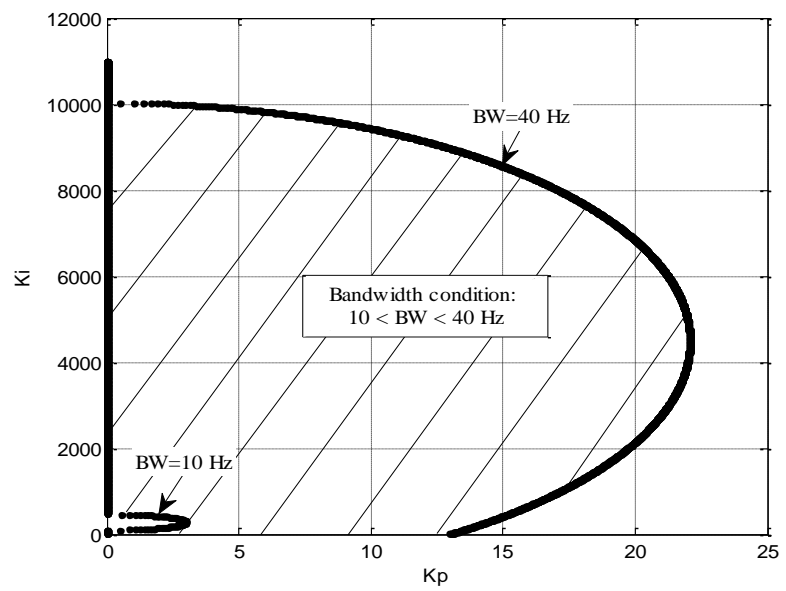

Figure 10

The stabilized area for $10<B W<40 \mathrm{~Hz}$

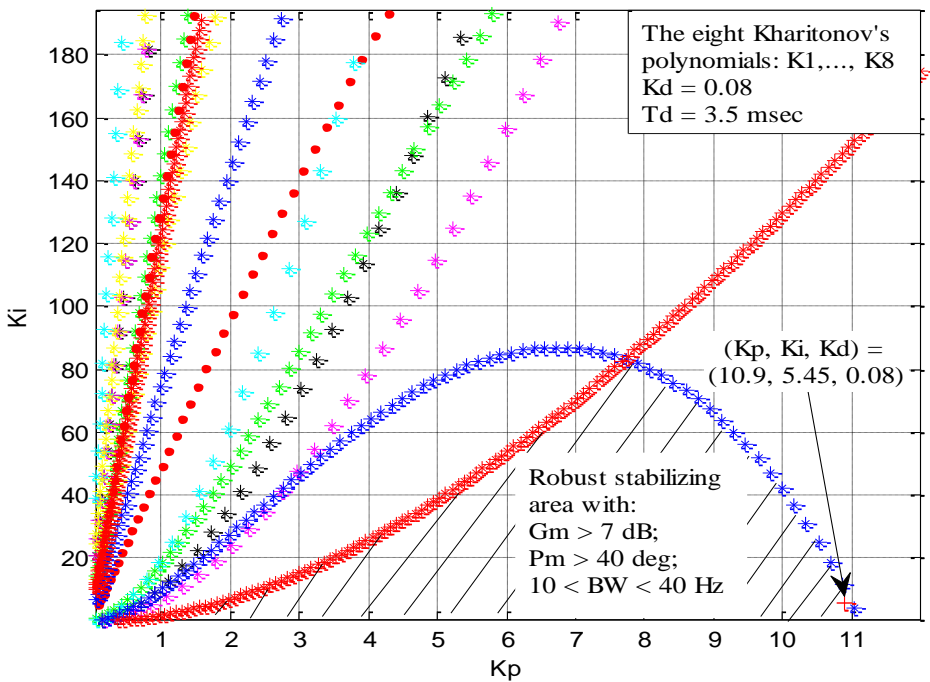

Figure 11

The stabilized lapped area for $P_{m} \geq 40^{\circ}, G_{m} \geq 7 \mathrm{~dB}$, and $10<B W<40 \mathrm{~Hz}$

The PID controller coefficients should be selected from the stabilized area shown in Figure 11, so that the desired performance requirements of the EMA system are attained. 
The selected PID controller is: $\left(K_{p}, K_{i}, K_{d}\right)=(10.9,5.45,0.08)$. To affirm the method validation, the gain and phase margins for the polynomials family with the designed controller for all the edges uncertainties were extracted and tabulated in Table 2. It is noted that the constraint of required margins $P_{m} \geq 40^{\circ}$ and $G_{m} \geq 7 \mathrm{~dB}$ is verified.

Table 2

Margins for polynomials family with controller $(10.9,5.45,0.08)$

\begin{tabular}{|c|l|l|l|l|}
\hline \multicolumn{1}{|c|}{$K$} & \multicolumn{1}{|c|}{$q_{1}$} & \multicolumn{1}{|c|}{$q_{2}$} & $G_{m}(d B)$ & $P_{m}($ deg $)$ \\
\hline 949.7 & \multirow{2}{*}{197.6} & 90.5 & 16.4 & 85.5 \\
\cline { 3 - 5 } & & 35 & 16.4 & 85.2 \\
\cline { 3 - 5 } & \multirow{2}{*}{90.4} & 90.5 & 14.6 & 59.2 \\
\cline { 3 - 5 } & & 35 & 14.6 & 59.1 \\
\hline \multirow{3}{*}{12.3} & \multirow{2}{*}{197.6} & 90.5 & 21.7 & 87.8 \\
\cline { 3 - 5 } & & 35 & 21.7 & 87.3 \\
\cline { 3 - 5 } & \multirow{2}{*}{90.4} & 90.5 & 20 & 69.4 \\
\cline { 3 - 5 } & & 35 & 20 & 69 \\
\hline
\end{tabular}

\subsection{Stability and Performance Robustness Analysis}

Figure 12 is the vivid illustration of the nominal closed-loop system analysis with maximum time delay, which indicates its high robustness with $17.6 d B$ gain margin and $79.7 \mathrm{deg}$ of phase margin with bandwidth $12.9 \mathrm{~Hz}$.

The worst-case analysis (peak-over-frequency type) shows a degradation of the gain and phase margins to a mere $10 \mathrm{~dB}$ and $54.9 \mathrm{deg}$, which takes place at frequency $24.5 \mathrm{~Hz}$. However, the margins are still acceptable compared to the system's requirements. In addition, the frequency at which the worst-case takes place $(24.5 \mathrm{~Hz})$ is somewhat larger than the system's bandwidth. 


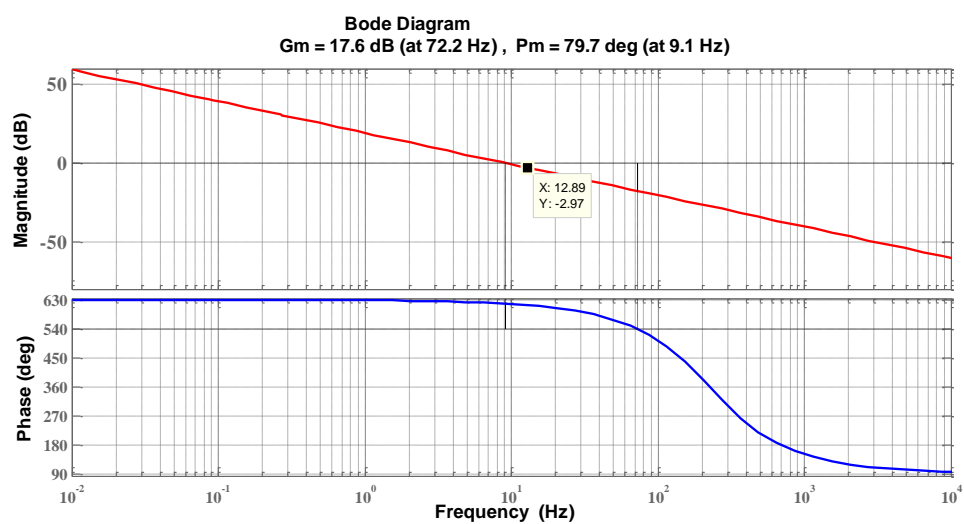

Figure 12

Bode diagram of nominal system with controller $(10.9,5.45,0.08)$

The sensitivity function is a measure of closed-loop performance for the EMA feedback control system. In the time domain, the sensitivity function indicates how well a step disturbance can be rejected. The uncertain sensitivity function $\mathrm{S}$ was calculated and the Bode magnitude plots for the nominal and worst-case values of the sensitivity function were compared, as shown in Figure 13.

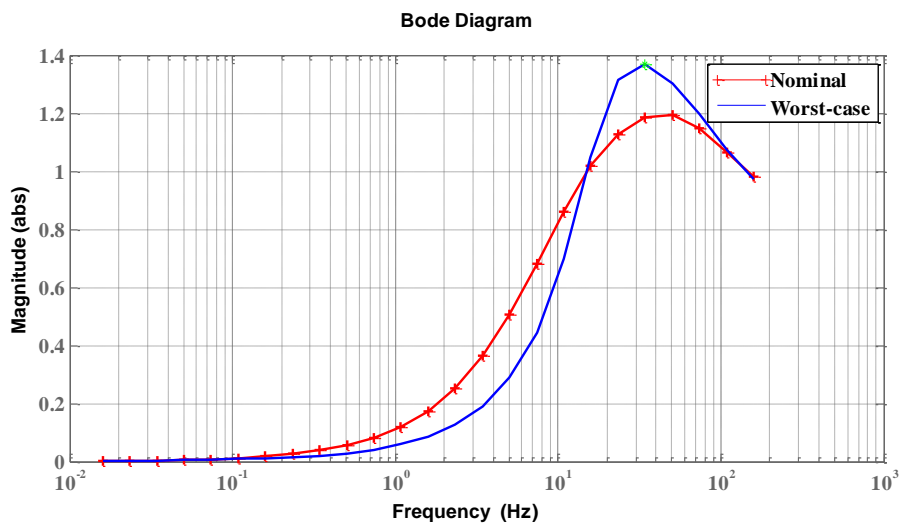

Figure 13

Bode magnitude plot of the nominal and worst-case sensitivity

It is observed that the sensitivity magnitude exceeds one at $(15.1 \mathrm{~Hz})$ which is more than the bandwidth of EMA system with the designed controller $(12.9 \mathrm{~Hz})$. The critical value of frequency at which maximum gain occurs $(34 \mathrm{~Hz})$ as shown in Figure13, with Lower bound $2.714 \mathrm{~dB}$ and Upper bound $2.722 \mathrm{~dB}$. The sensitivity function step response, is plotted to observe the variability in disturbance rejection characteristics, as illustrated in Figure 14. 


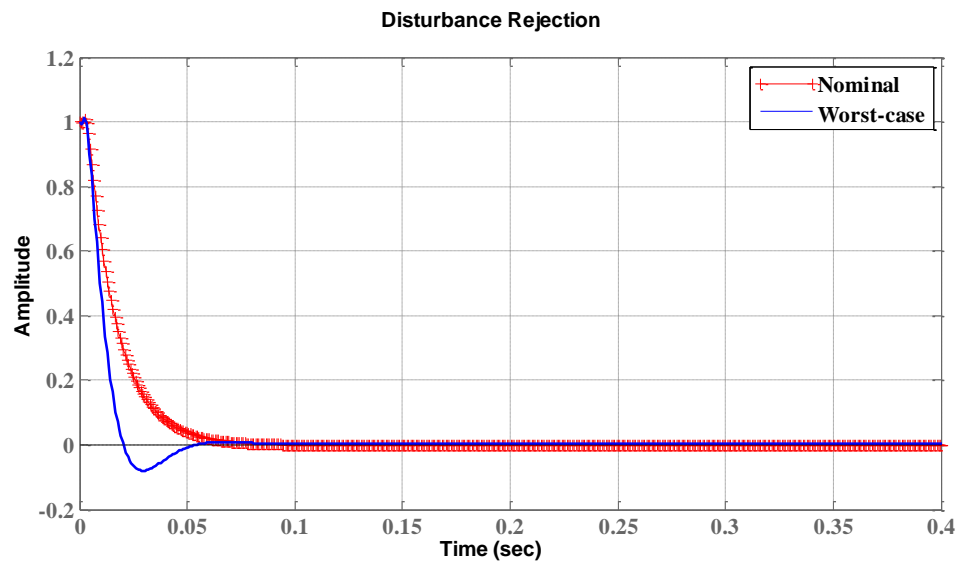

Figure 14

Step response of the nominal and worst-case sensitivity

Since it is enough to test the origin exclusion for $\omega \leq \omega_{c}$ (the crossover frequency which is less than $100 \mathrm{rad} / \mathrm{sec}$ in the studied system), the rectangles were plotted for frequencies $\omega<200 \mathrm{rad} / \mathrm{sec}$ in Figure 15.

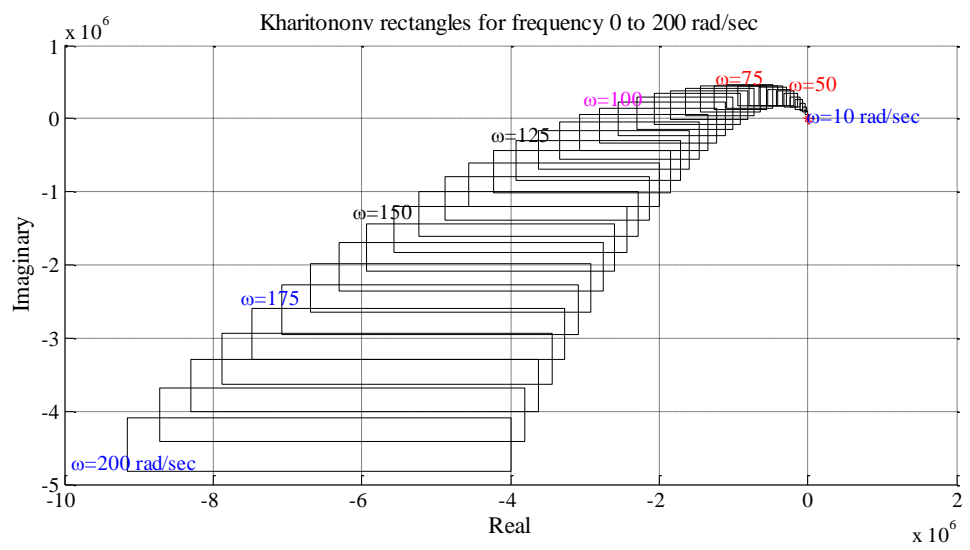

Figure 15

Kharitonov rectangles for the EMA system

As shown in Figure 15, the origin is excluded from the Kharitonov's rectangles, which affirms that the EMA closed loop system is robustly stable. 


\section{Robust Controller Validation}

The EMA system, with the new robust controller, will be validated by Bials's test and by comparing its performance with the original EMA performance.

\subsection{Validation by Bialas' Test}

Suppose that the polynomials $p_{1}$ and $p_{2}$ are strictly Hurwitz with their leading coefficient nonnegative and the remaining coefficients positive. Let $P_{1}$ and $P_{2}$ be their Hurwitz matrices, and define the matrix:

$W=-P_{1} P_{2}^{-1}$

Then each of polynomials

$p=\lambda p_{1}+(1-\lambda) p_{2}, \lambda \in[0,1]$

is strictly Hurwitz iff the real eigenvalues of $W$ all are strictly negative.

Remark: the Hurwitz matrix $Q$, for the polynomial $q(s)$, is defined as follows

$q(s)=q_{0} s^{n}+q_{1} s^{n-1}+q_{2} s^{n-2} \ldots+q_{n} Q=\left[\begin{array}{cccccc}q_{1} & q_{3} & q_{5} & \cdots & \cdots & \cdots \\ q_{0} & q_{2} & q_{4} & \cdots & \cdots & \cdots \\ 0 & q_{1} & q_{3} & q_{5} & \cdots & \cdots \\ 0 & q_{0} & q_{2} & q_{4} & \cdots & \cdots \\ 0 & 0 & q_{1} & q_{3} & q_{5} & \cdots \\ \ldots & \cdots & \cdots & \ldots & \cdots & \cdots\end{array}\right]$

The designed controller by Kharitonov's theorem should be submitted to the Bialas' test at its 12 exposed edges.

For the first exposed edge: the two polynomials after numerical substitution are

$p_{11}(s)=2 s^{3}+262.78 s^{2}+11239 s+5584.3$,

$p_{12}(s)=2 s^{3}+262.78 s^{2}+11349 s+5584.3$

Which are both Hurwitz . The Hurwiz matrices of these two polynomials are:

$P_{11}=\left[\begin{array}{ccc}262.78 & 5584.3 & 0 \\ 2 & 11239 & 0 \\ 0 & 262.78 & 5584.3\end{array}\right], \quad P_{12}=\left[\begin{array}{ccc}262.78 & 5584.3 & 0 \\ 2 & 11349 & 0 \\ 0 & 262.78 & 5584.3\end{array}\right]$

The eigenvalues of $W_{1}=-P_{11} P_{12}^{-1}$ are $-1,-0.9903$, and -1 . They are all real and negative. Hence, by Bials' test, the EMA system is stable on the first edge.

Similarly, the other 11 were checked and the eigenvalues of $W_{i}$ for $i=2 \ldots 12$ are all real negative. Another example, the eigenvalues of $W_{12}=-P_{121} P_{122}^{-1}$ are $0.5394,-0.5410$, and -0.8718 . So, by the edge theorem, the system is robustly stable for the uncertainties under consideration. 


\subsection{Performance Validation}

Finally, the step responses of the EMA system with the designed robust controller and the identified original one were plotted (as illustrated in Figure 16).

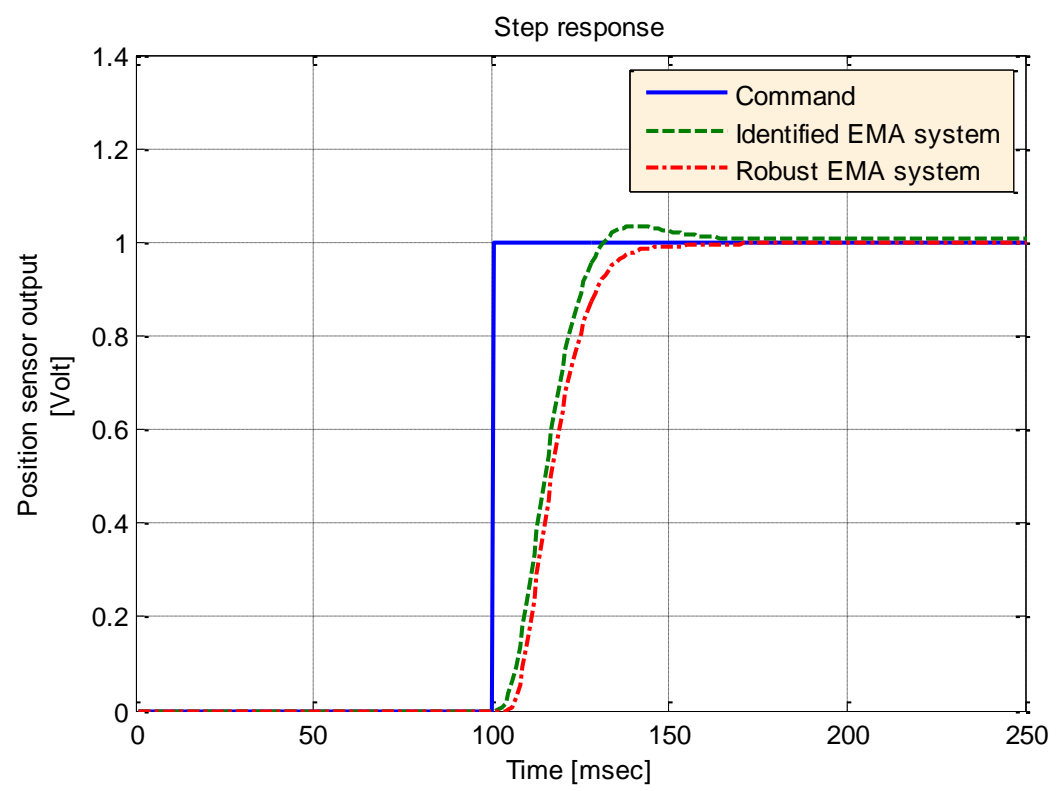

Figure 16

Step responses for EMA systems

The main characteristics of the EMA systems are shown in Table 3. The new designed EMA system, in addition to its robustness to parametric uncertainties and margins achievement, has no overshoot. In addition, in spite of small degradation in rise and settling times, they are still within the acceptable ranges.

Table 3

Characteristics of original and robust EMA systems

\begin{tabular}{|l|c|c|c|c|}
\hline Model & $\begin{array}{c}\mathrm{t}_{\mathrm{r}(0 \sim 90 \%)} \\
(\mathrm{msec})\end{array}$ & $\begin{array}{c}\mathrm{t}_{\mathrm{s}} \\
(\mathrm{msec})\end{array}$ & $\begin{array}{c}\mathrm{t}_{\mathrm{p}} \\
(\mathrm{msec})\end{array}$ & $\begin{array}{c}\text { Overshoot } \\
(\%)\end{array}$ \\
\hline Identified original EMA & 25 & 46 & 39.5 & 2.6 \\
\hline Robust EMA & 30 & 48 & -- & 0 \\
\hline
\end{tabular}

A low pass filter (LPF) is added to the derivative path of the PID controller in order to pass only low frequency gain and attenuate the high frequency one.

$$
\operatorname{LPF}(s)=\frac{1}{0.001 s+1}
$$


To attain the last results, the EMA system with the designed robust controller secures the robust stability over the uncertainties intervals and attains the required margins $\left(P_{m} \geq 40^{\circ}, G_{m} \geq 7 d B\right)$ with an acceptable bandwidth. In time domain, it has no overshoots and no steady-state errors. Therefore, all the required specifications shown in Table 1 are accomplished.

\section{Conclusions}

A novel approach was proposed, to graphically design a robust PID controller, for a parametric uncertain system with time delay constrained with gain and phase margins conditions. It was then applied to an EMA system, the designed controller ensured robust stability, while shaping the performance in a desired fashion. Finally, in order to validate its practicality, the robust EMA system was compared with the identified EMA system. In addition, to its robustness, the robust EMA system proved better dynamic characteristics than the original.

\section{References}

[1] C. Gerada, and K. J. Bradley: Integrated PM Machine Design for an Aircraft EMA, IEEE Transactions on Industrial Electronics, Vol. 55, No. 9, 2008, pp. 3300-3306

[2] D. Howe: Magnetic Actuators, Sensors and Actuators, Vol. 81, 2000, pp. 268-274

[3] J. Liscouët, J.-C. Maré, and M. Budinger: An Integrated Methodology for the Preliminary Design of Highly Reliable Electromechanical Actuators: Search for Architecture Solutions, Aerospace Science and Technology, Vol. 22, 2012, pp. 9-18

[4] E. N. Gonçalves, R. M. Palhares, and R. H. C. Takahashi: $\mathrm{H}_{2} / \mathrm{H}_{\infty}$ Robust PID Synthesis for Uncertain Systems, Proceedings of the $45^{\text {th }}$ IEEE conference on Decision \& Control, San Diego, CA, USA, December 13-15, 2006, pp. 4375-4380

[5] T. Yamamoto, T. Ueda, H. Shimegi, T. Sugie, S. Tujio, and T. Ono: A Design Method of Robust Controller and Its Application to Positioning Servo, JSME International Journal, Series III, Vol. 33, No. 4, 1990, pp. 649-654

[6] H. Lu, Y. Li, and C. Zhu: Robust Synthesized Control of Electromechanical Actuator for Thrust Vector System in Spacecraft, J. Computers and Mathematics with Applications, Vol. 64, Issue 5, 2012, pp. 699-708

[7] S. E. Lyshevski, R. D. Colgren, and V. A. Skormin: High Performance Electromechanical Direct-Drive Actuators for Flight Vehicles, Proceedings of the American Control Conference, Arlington, VA, June 25-27, 2001, pp. $1345-1350$ 
[8] S. E. Lyshevski: Electromechanical Flight Actuators for Advanced Flight Vehicles, IEEE Transactions on Aerospace and Electronic Systems Journal, Vol. 35, No. 2, 1999, pp. 511-518

[9] M. Ristanović, Ž. Ćojbašić, and D. Lazić: Intelligent Control of DC Motor Driven Electromechanical Fin Actuator, J. Control Engineering Practice, Vol. 20, 2012, pp. 610-617

[10] C. Yoo, Y. Lee, and S. Lee: A Robust Controller for an Electro-Mechanical Fin Actuator, Proceeding of the American Control Conference, Boston, Massachusetts, June 30-July 2, 2004, pp. 4010-4015

[11] J. Dawes, L. Ng, R. Dorf, and C. Tam: Design of Deadbeat Robust Systems, Glasgow, UK, 1994, pp. 1597-1598

[12] R. Toscano: A Simple Robust PI/PID Controller Design via Numerical Optimization Approach, J. of Process Control, Vol. 15, 2005, pp. 81-88

[13] D. Valério, J. Costa: Tuning of Fractional Controllers Minimising H2 and $\mathrm{H}_{\infty}$ Norms, Acta Polytechnica Hungarica, Vol. 3, No. 4, 2006, pp. 55-70

[14] R. Bréda, T. Lazar, R. Andoga, and L. Madarász: Robust Controller in the Structure of Lateral Control of Maneuvering Aircraft, Acta Polytechnica Hungarica, Vol. 10, No. 5, 2013, pp. 101-124

[15] N. Tan: Computation of Stabilizing PI-PD Controllers, International Journal of Control, Automation, and Systems, 7(2), 2009, pp. 175-184

[16] Y. J. Huang and Y. J. Wang: Robust PID Tuning Strategy for Uncertain Plants Based on the Kharitonov Theorem, ISA Transactions, Vol. 39, 2000, pp. 419-431

[17] P. C. Krause, O. Wasynczuk, and S. D. Sundhoff: Analysis of Electric Machinery and Drive Systems, IEEE press, 2002

[18] K.-K. Shyu, and Y.-Y. Lee: Identification of Electro-Mechanical Actuators within Limited Stroke, Journal of Vibration and Control, Vol. 16(12), 2010, pp. 1737-1761

[19] S. P. Bhattacharyya, H. Chapellat, and L. H. Keel: Robust Control: The Parametric Approach, Prentice Hall, 1995 